Supporting Information for:

\title{
Novel Polyphenylenes Containing Phenol Substituted Oxadiazole Moieties as Fluorescent Chemosensors for Fluoride Ion
}

Gang Zhou, Yanxiang Cheng, Lixiang Wang, Xiabin Jing and Fosong Wang

Contribution from the State Key Laboratory of Polymer Physics and Chemistry, Changchun Institute of Applied Chemistry, Chinese Academy of Sciences, Changchun 130022, P.R. China

\section{Contents:}

1. Fluorescence and absorption spectra of polymers P2-P4 before their demethylation.

2. Fluorescence and absorption spectra of polymers P6-P8 before their demethylation.

3. Fluorescence and absorption spectra of polymers P2-P4 $(5 \mu \mathrm{M})$ in chloroform

4. Fluorescence and absorption spectra of polymers P6-P8 $(5 \mu \mathrm{M})$ in chloroform

5. Fluorescence spectra of polymer $\mathbf{P} 3(5 \mu \mathrm{M})$ in different solvents

6. Fluorescence titration-general procedure.

7. Absorption spectra for the titration of $\mathbf{P 3}$ with $\mathrm{Bu}_{4} \mathrm{NF}$.

8. Fluorescence spectra for the titration of P2, P4, P6-P8 with $\mathrm{Bu}_{4} \mathrm{NF}$.

9. Fluorescence and absorption spectra for the titration of $\mathbf{P 1}+\mathbf{P 5}$ with $\mathrm{Bu}_{4} \mathrm{NF}$. 
1. Fluorescence and absorption spectra of polymers P2-P4 before their demethylation.
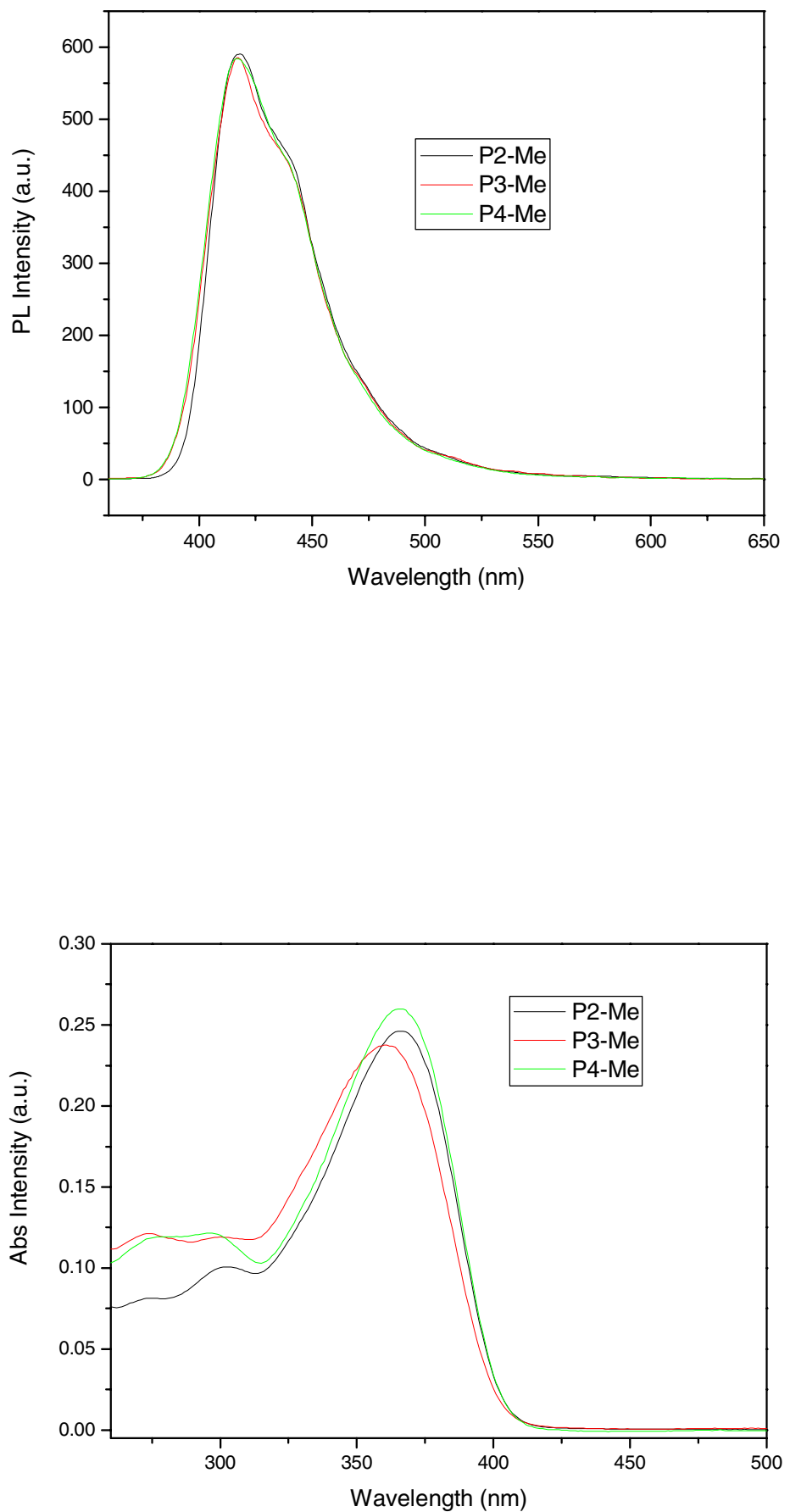
2. Fluorescence and absorption spectra of polymers P6-P8 before their demethylation.
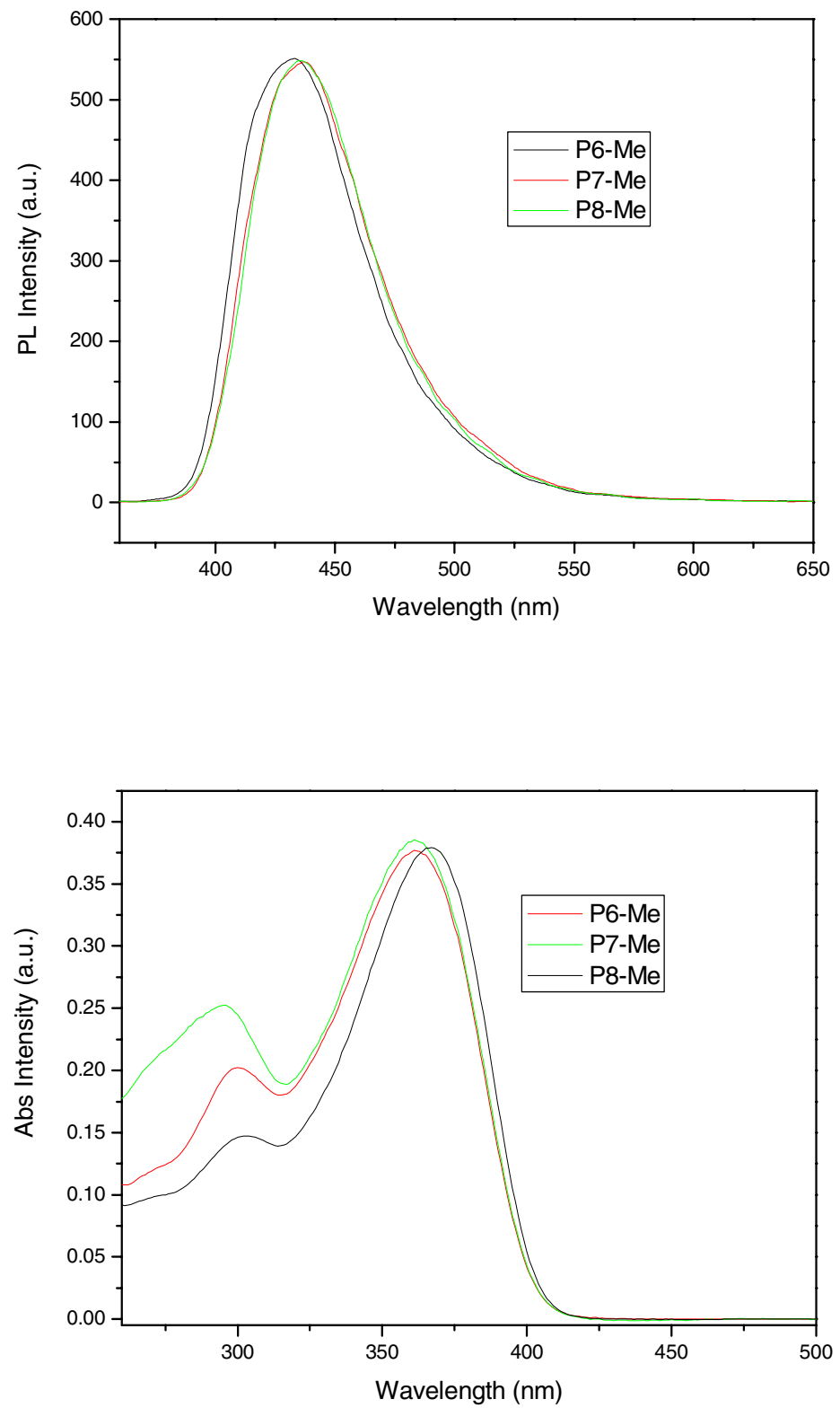
3. Fluorescence and absorption spectra of polymers P2-P4 $(5 \mu \mathrm{M})$ in chloroform
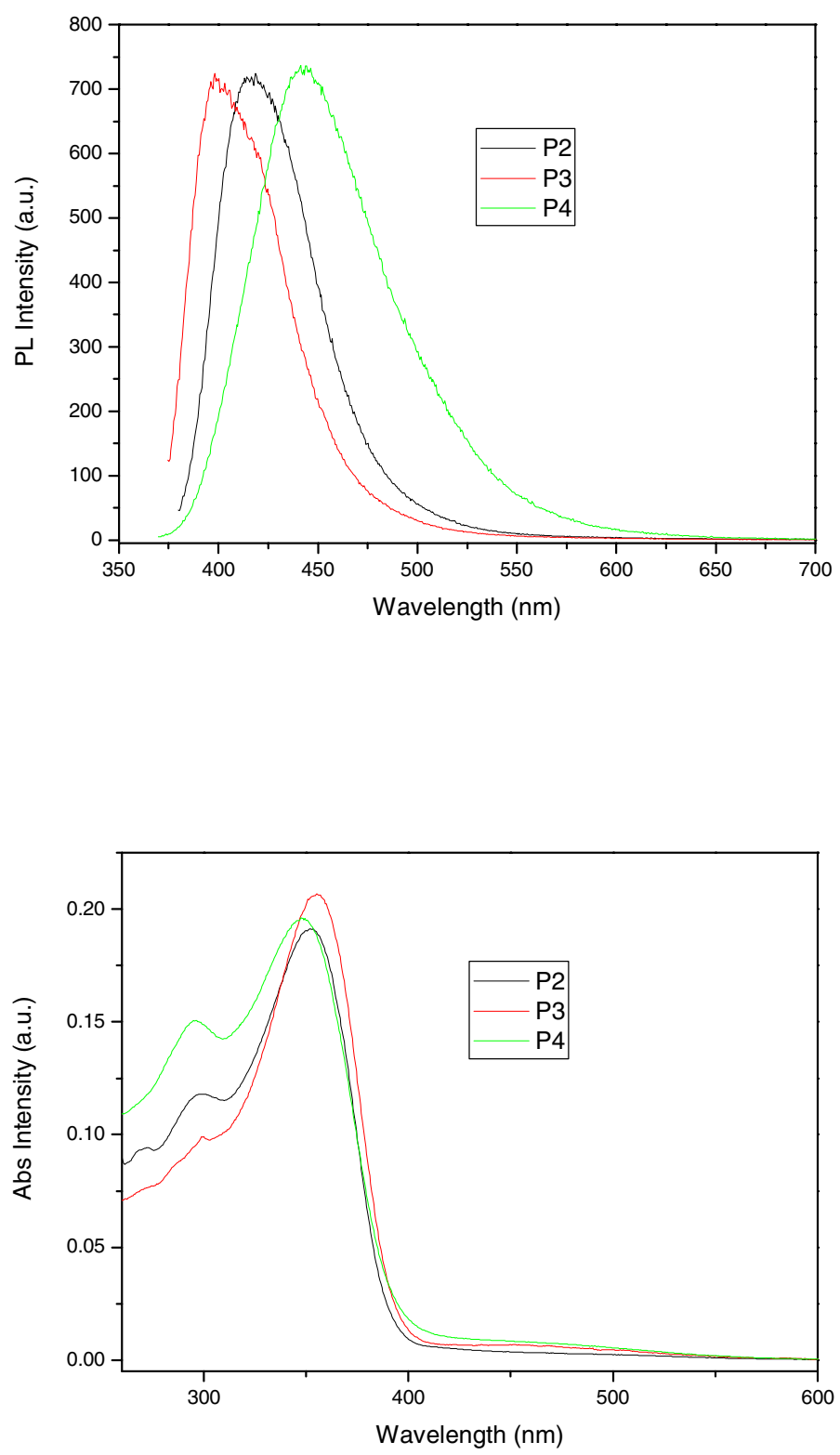
4. Fluorescence and absorption spectra of polymers P6-P8 $(5 \mu \mathrm{M})$ in chloroform
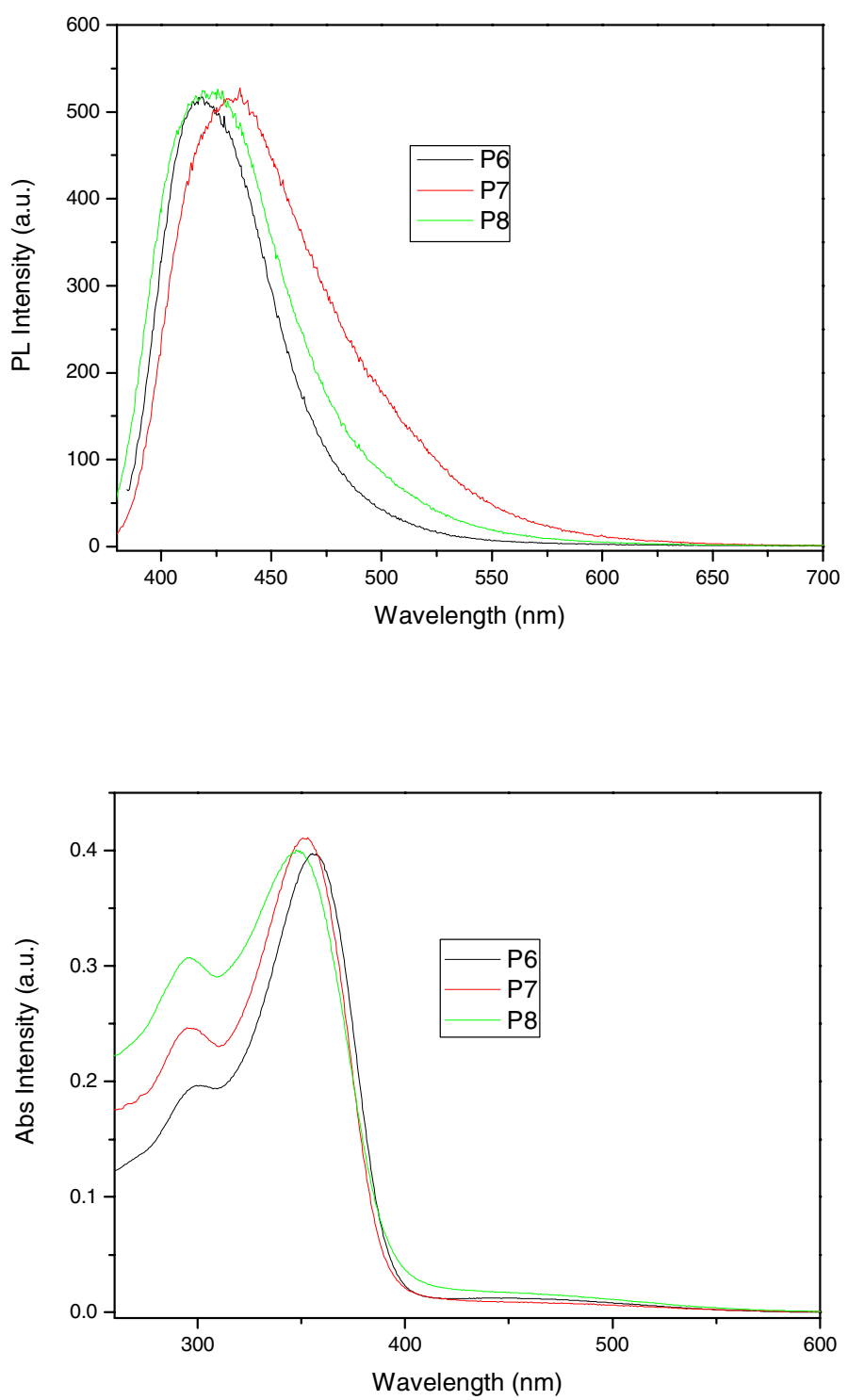
5. Fluorescence spectra of polymer $\mathbf{P 3}(5 \mu \mathrm{M})$ in different solvents

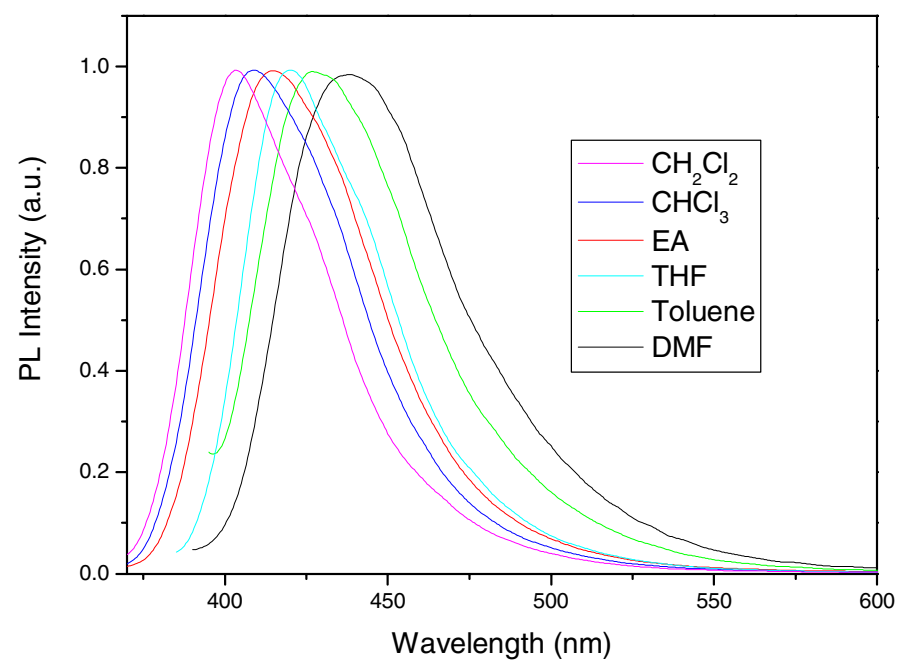


6. Fluorescence titration-general procedure:

Fluorescence spectra were obtained on a Perkin-Elmer LS 50B luminescence spectrometer with Xenon discharge lamp excitation. Measurements of Ultraviolet-Visible absorption spectra were carried out on Perkin-Elmer Lambda 35 UV/Vis spectrometer. Solution of polymers P1-P10 were dilute at a concentration of $5 \times 10^{-6} \mathrm{M}$ (the concentration of polymers is based on the moles of the repeated unit) in $\mathrm{CHCl}_{3}$. Solutions of tetrabutylammonium anionic salts with the concentration of typically $1 \times 10^{-3} \mathrm{M}$ were prepared by successive dilution. Fluorescence titration was carried out by sequentially adding $0.1 \mathrm{~mL}$ aliquots of anions solutions in $\mathrm{CHCl}_{3}$ to 5.00 $\mathrm{ml}$ of the polymer solutions, and $\mathrm{CHCl}_{3}$ was added to acquire $10.00 \mathrm{ml}$ solutions. The solutions were stirred for 5 min prior to obtaining fluorescence spectra. 
7. Absorption spectra for the titration of $\mathbf{P 2}$ with $\mathrm{Bu}_{4} \mathrm{NF}$.

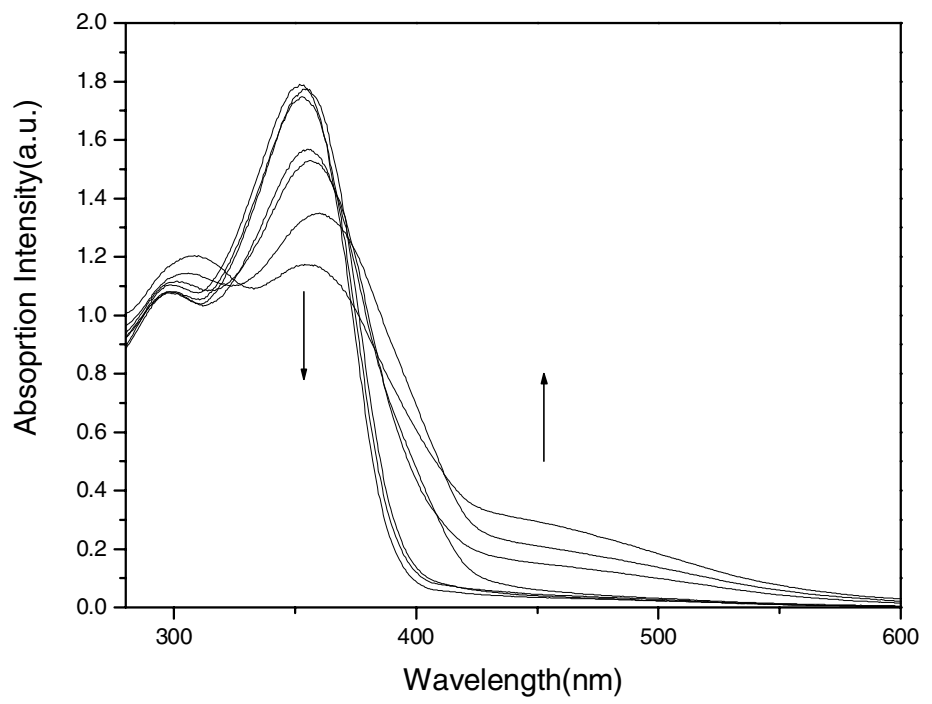


8. Fluorescence spectra for the titration of $\mathbf{P 2}, \mathbf{P 4}, \mathbf{P 6}-\mathbf{P 8}$ with $\mathrm{Bu}_{4} \mathrm{NF}$.

Fluorescence emission for titration of $\mathbf{P 2}$ with $0-100 \mu \mathrm{M} \mathrm{Bu}_{4} \mathrm{NF}$ in $\mathrm{CHCl}_{3}$

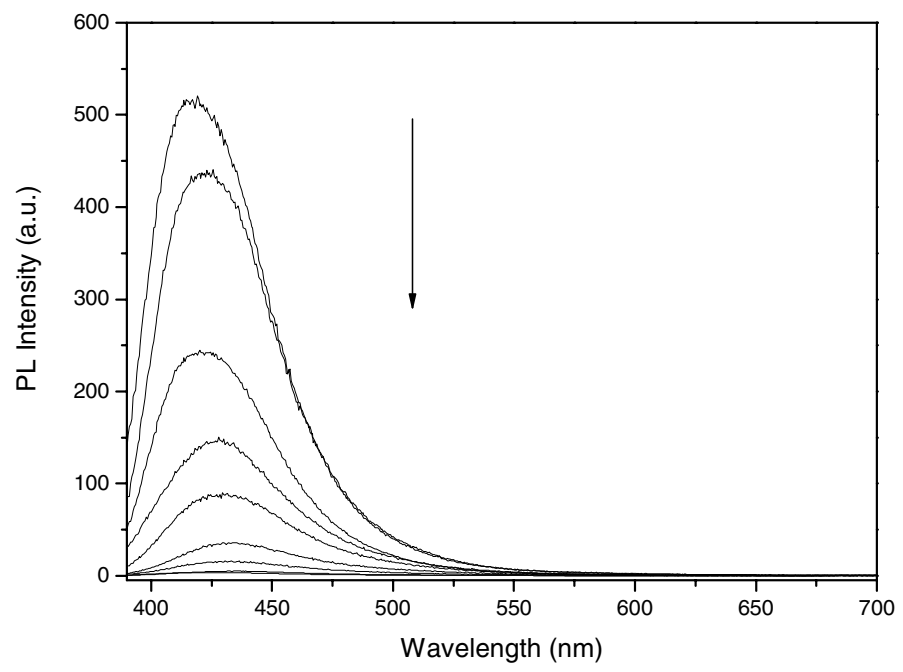

Fluorescence emission for titration of $\mathbf{P 4}$ with $0-100 \mu \mathrm{M} \mathrm{Bu}_{4} \mathrm{NF}$ in $\mathrm{CHCl}_{3}$

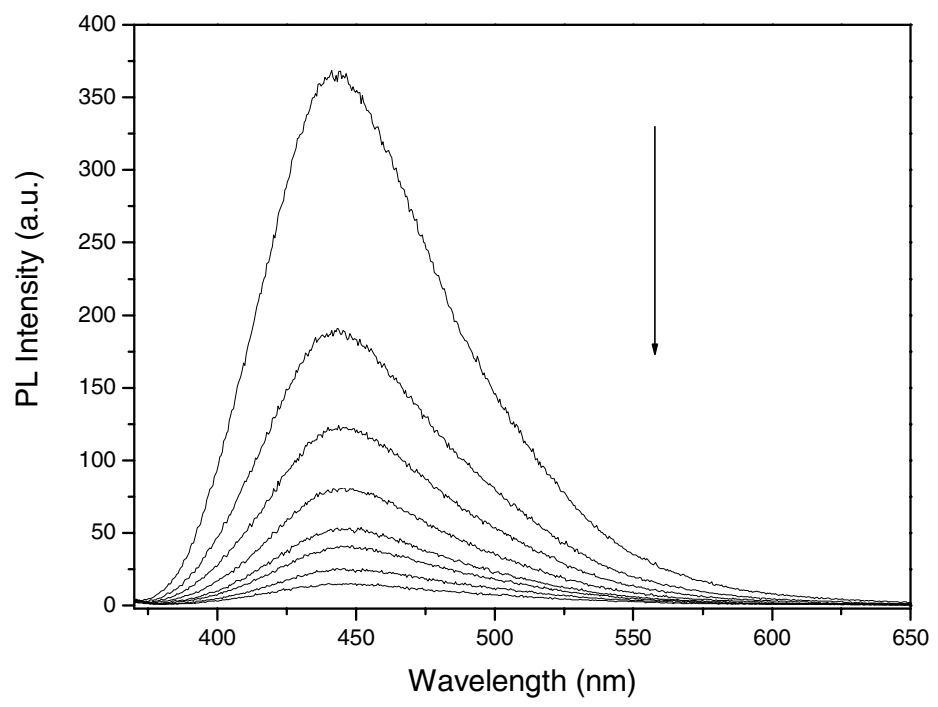


Fluorescence emission for titration of $\mathbf{P 6}$ with 0-100 $\mu \mathrm{M} \mathrm{Bu}_{4} \mathrm{NF}$ in $\mathrm{CHCl}_{3}$

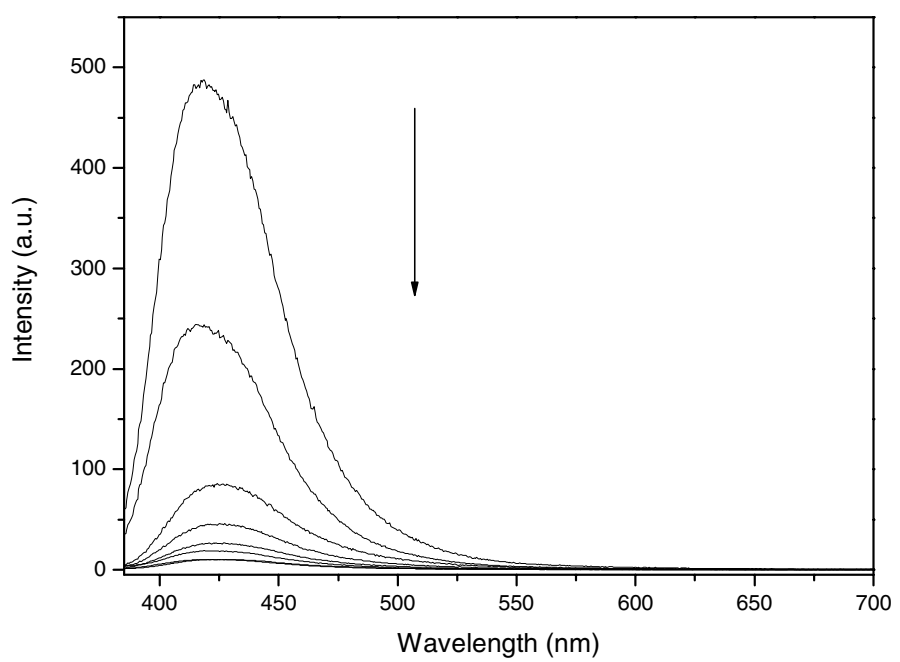

Fluorescence emission for titration of $\mathbf{P 7}$ with 0-100 $\mu \mathrm{M} \mathrm{Bu}_{4} \mathrm{NF}$ in $\mathrm{CHCl}_{3}$

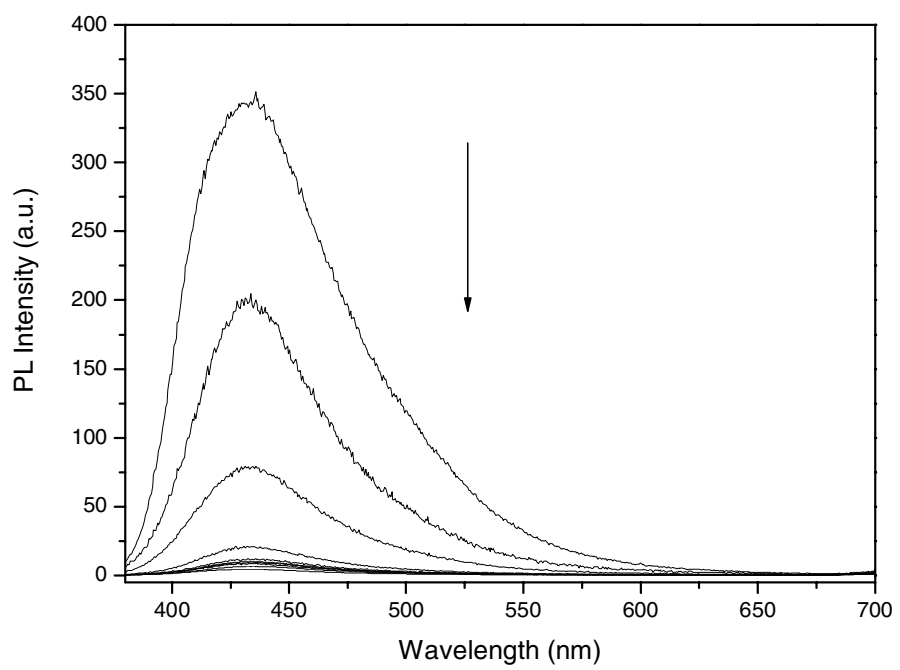


Fluorescence emission for titration of $\mathbf{P 8}$ with 0-100 $\mu \mathrm{M} \mathrm{Bu}_{4} \mathrm{NF}$ in $\mathrm{CHCl}_{3}$

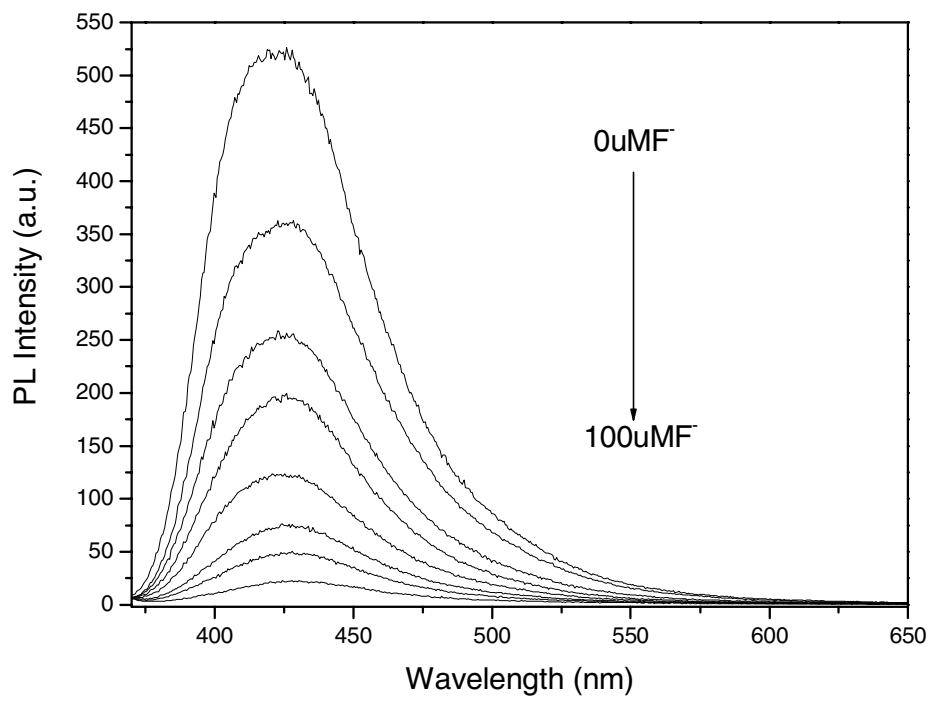


9. Absorption and fluorescence spectra for the titration of $\mathbf{P 1}+\mathbf{P 5}$ with $\mathrm{Bu}_{4} \mathrm{NF}$.

Fluorescence emission for titration of $\mathbf{P 1}+\mathbf{P 5}$ with $0-100 \mu \mathrm{M} \mathrm{Bu}_{4} \mathrm{NF}$ in $\mathrm{CHCl}_{3}$

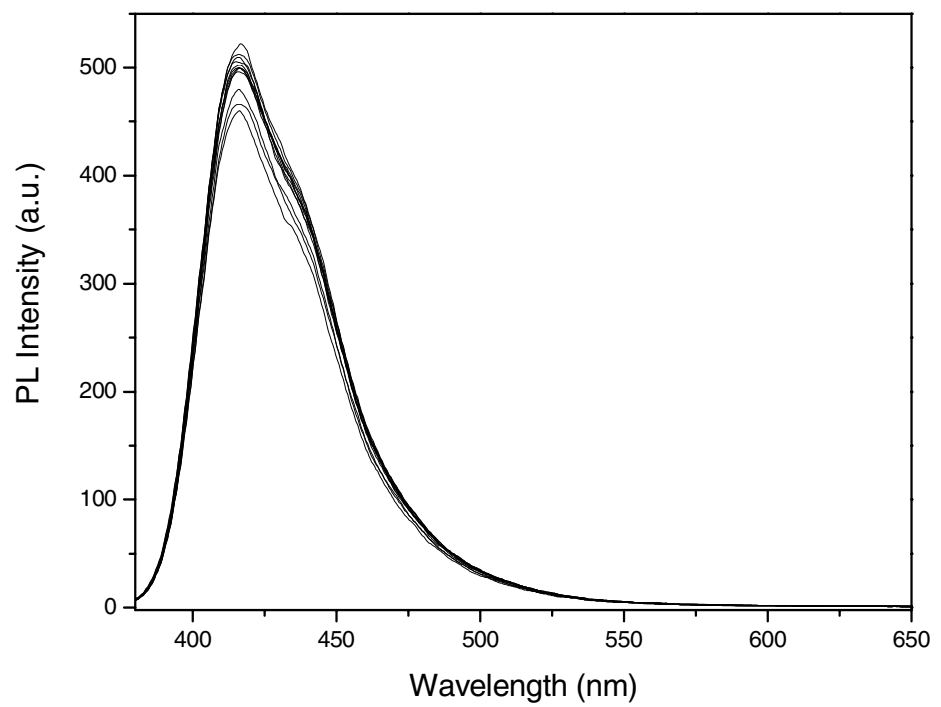

Absorption spectra for titration of $\mathbf{P 1}+\mathbf{P 5}$ with $0-100 \mu \mathrm{M} \mathrm{Bu}_{4} \mathrm{NF}$ in $\mathrm{CHCl}_{3}$

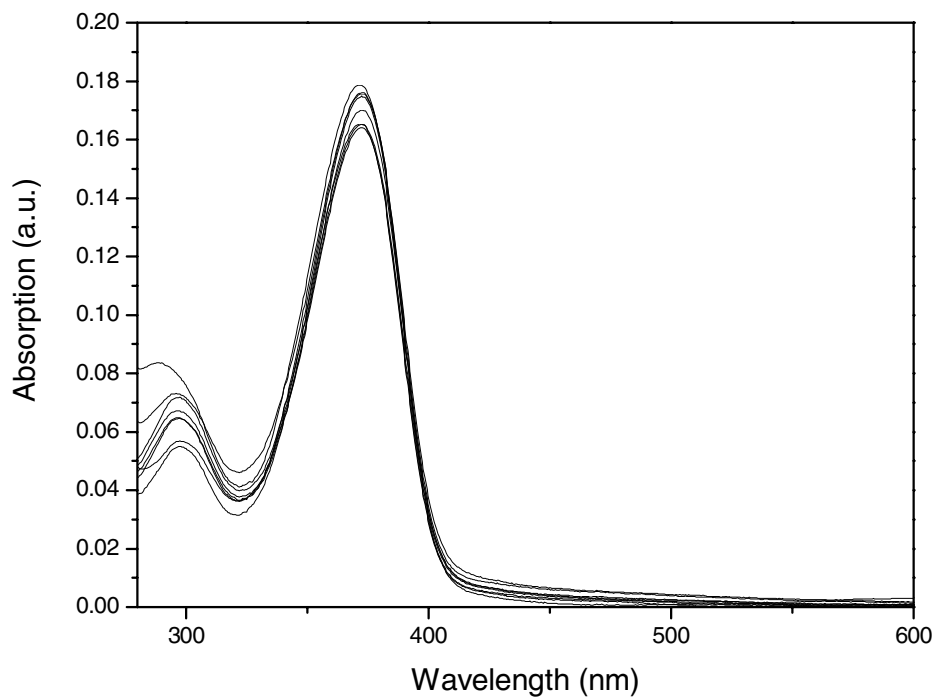

